\title{
Safe Transcatheter Aortic Valve Replacement in a Patient with a Highly Mobile Aortic Valve Mass
}

\author{
Brian B. Agbor-Etang, MD ${ }^{1,2}$ Ashis Mukherjee, MD $^{1,2}$ Prabhdeep S. Sethi, MD ${ }^{1,2}$ Ramdas G. Pai, MD ${ }^{1,2}$ \\ 1 Division of Cardiology, Department of Internal Medicine, University \\ of California, School of Medicine, Riverside, California \\ 2 Division of Cardiology, Department of Internal Medicine, \\ St Bernadine Medical Center, San Bernardino, California

\begin{abstract}
Address for correspondence Ramdas G. Pai, MD, Department of Medicine, University of California School of Medicine, 900 University Avenue, SOM Education Building \#2619, Riverside, CA 92521 (e-mail: Ramdas.pai@ucr.edu).
\end{abstract}

Int J Angiol 2018;27:174-176.

\begin{abstract}
Keywords

- cardiac

- aortic valve

Some cardiac valve masses may have embolic potential with worrisome consequences. We describe the dilemmas of and solutions for a highly mobile papillary fibroelastoma on the aortic valve in a nonsurgical patient undergoing transcatheter aortic valve replacement. It was performed safely. The potential strategies to minimize the risk of embolization are discussed.
\end{abstract}

Papillary fibroelastoma is a common cardiac tumor commonly attached to the valves ${ }^{1}$ with an embolic potential. We describe a case where it was attached to the aortic leaflet edge of a patient scheduled to undergo transcatheter aortic valve replacement (TAVR).

\section{Case}

An 86-year-old male with a history of hypertension, diabetes mellitus, and chronic kidney disease presented with dyspnea and chest heaviness on minimal effort. Echocardiogram revealed severe aortic stenosis with a valve area $0.7 \mathrm{~cm}^{2}$, mean gradient of $45 \mathrm{~mm} \mathrm{Hg}$, moderate left ventricular hypertrophy, and ejection fraction of $70 \%$. Due to frailty and elevated surgical risk (STS score of 9.8), TAVR was recommended by the heart valve team as the patient was deemed nonsurgical. However, the transesophageal echocardiogram (TEE) during TAVR showed a highly mobile, pedunculated mass measuring $\sim 0.9 \times 0.7 \mathrm{~mm}$, consistent with a fibroelastoma, attached to the aortic leaflet edge (-Fig. 1). The embolic potential during TAVR was deemed to be high because of its location, size, and mobility.

\section{Decision Making}

We, therefore, adopted the following strategies to minimize embolic risk: (1) The most worrisome risk was embolization into either of the coronaries or cerebral circulation, with the right brachiocephalic artery being the most likely target. A right carotid filter was used for cerebral protection. (2) No aortic valve predilation was performed. (3) The aortic valve was sized and crossed under TEE guidance. We did not use any coronary protection.

The patient also had renal dysfunction, not on dialysis; hence, preprocedural computed tomography planning could not be done. Aortic annulus sizing was performed using three-dimensional TEE and sizing of the peripheral vessels was achieved with the use of intravascular ultrasound.

TAVR was performed with $26 \mathrm{~mm}$ Sapien 3 valve via transfemoral approach. The mass got wedged between the valve and aortic wall without any embolization (-Fig. 2). To
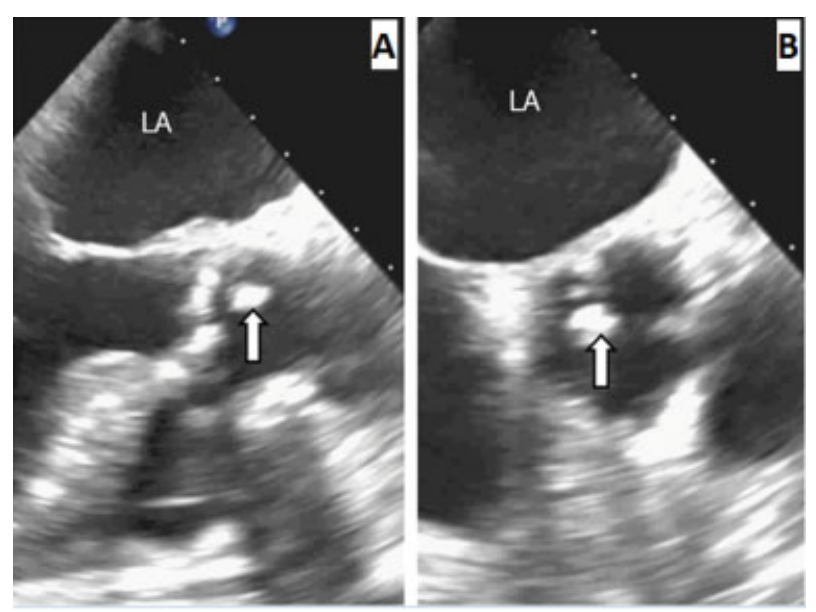

Fig. 1 Aortic valve long (A) and short axis (B) views. Arrow points to mobile, pedunculated mass on aortic valve leaflet edge consistent with a fibroelastoma. Abbreviation: LA, left atrium. published online

May 17, 2018
Copyright $\odot 2018$ by Thieme Medical Publishers, Inc., 333 Seventh Avenue, New York, NY 10001, USA. Tel: +1(212) 584-4662.
DOI https://doi.org/ $10.1055 / \mathrm{s}-0038-1639354$. ISSN 1061-1711. 


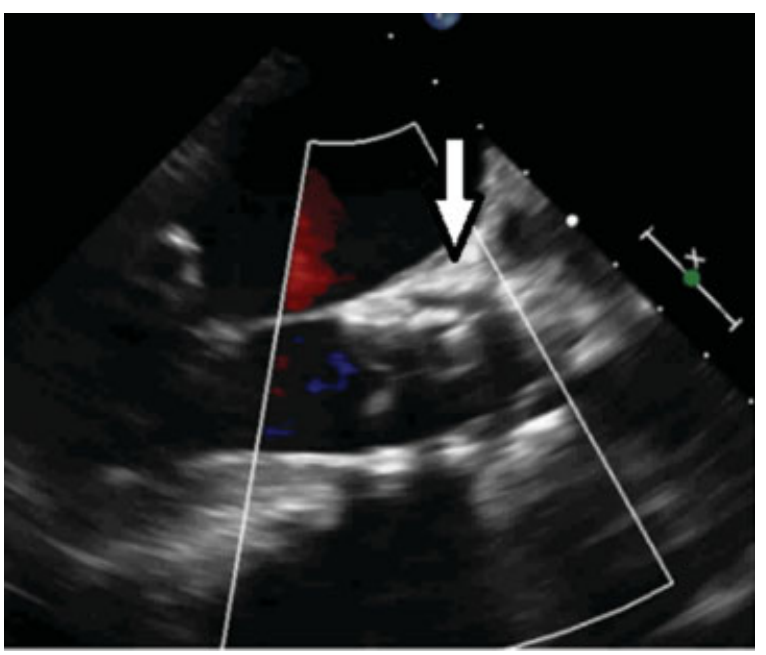

our knowledge, this is the first case where TAVR was performed successfully in a patient with mobile aortic fibroelastoma using carotid protection to minimize stroke risk.

\section{Conclusion}

Cerebral and/or coronary protection may be advisable during TAVR when there is a mobile aortic mass. It may be advisable to skip aortic valve predilation in such cases.

\section{References}

1 Edwards FH, Hale D, Cohen A, Thompson L, Pezzella AT, Virmani R. Primary cardiac valve tumors. Ann Thorac Surg 1991;52(05): 1127-1131

Fig. 2 During transcatheter aortic valve replacement, the mass (arrow) got wedged between the prosthetic valve and aortic wall without any embolization. 